\title{
Effects of Atorvastatin on Nuclear Magnetic Resonance-Defined Lipoprotein Subclasses and Inflammatory Markers in Patients with Hypercholesterolemia
}

\author{
Katsunori Ikewaki ${ }^{1}$, Yoshio Terao ${ }^{2}$, Hideki Ozasa², Yoshinobu Nakada², Jun-ichiro Tohyama², Yae Inoue ${ }^{1}$, \\ and Michihiro Yoshimura²
}

${ }^{1}$ Division of Anti-Aging, Department of Internal Medicine, National Defense Medical College, Tokorozawa, Saitama, Japan

${ }^{2}$ Division of Cardiology, Department of Internal Medicine, Jikei University School of Medicine, Tokyo, Japan

\begin{abstract}
Aim: Information about the effects of HMG-CoA reductase inhibitor (statin) treatment on lipoprotein subclasses has been severely limited. Nuclear magnetic resonance (NMR) spectrometry has emerged as a new methodology to quantify lipoprotein subclass concentrations. In the present study, we attempted to evaluate the hypolipidemic effects of atorvastatin utilizing this method.

Methods: Twenty-six patients were administered with atorvastain $10 \mathrm{mg}$ daily for 4 weeks. Lipoprotein subclasses were measured by nuclear magnetic resonance (NMR) spectroscopy. Inflammation markers, including C-reactive protein (CRP), interleukin-6 (IL-6), and monocyte chemotactic protein-1 (MCP-1), were also determined.

Results: Additional to a marked reduction of LDL-C (-43\%), atorvastatin treatment significantly decreased TG, RLP-C, apoC-II, apoC-III, and apoE by $27 \%$, 49\%, 25\%, 15\%, and $28 \%$, respectively. NMR analysis revealed marked reductions of all LDL subclasses, resulting in a significant reduction of LDL particle number as well as an increase in LDL particle size. Further, some VLDL were decreased and HDL particle size was increased by atorvastatin. Among inflammation markers, MDA-LDL and IL-6 were marginally to significantly decreased.

Conclusion: In addition to a strong LDL-C lowering function, atorvastatin exerts beneficial effects on TG-rich lipoproteins and inflammation in hypercholesterolemic patients.
\end{abstract}

J Atheroscler Thromb, 2009; 16:51-56.

Key words; Atorvastatin, Nuclear magnetic resonance, Inflammatory marker

\section{Introduction}

An increased level of low-density lipoprotein (LDL) cholesterol (C) is an established risk factor for coronary heart disease (CHD). The clinical benefit of LDL-C-lowering therapy using HMG-CoA reductase inhibitors (statins) has been well documented, contributing to the management of dyslipidemia in the current guideline ${ }^{1)}$. At present, several statins varying in LDL-C-lowering potency are available, thus provid-

Address for correspondence: Katsunori Ikewaki, Division of Anti-Aging, Department of Internal Medicine National Defense Medical College 3-2 Namiki, Tokorozawa, Saitama 359-8513, Japan

E-mail: katsunorike@ndmc.ac.jp

Received: December 4, 2007

Accepted for publication: October 28, 2008 ing clinicians more options based on the target LDL-C level. Nonetheless, recent data have demonstrated limited success with respect to achieving the LDL-C target, in particular among high-risk patients ${ }^{2,3)}$, keeping pravastatin, a mild statin, as the standard drug for the treatment of hypercholesterolemic patients in Japan. Atorvastatin exerts a strong LDL-C-lowering effect and, together with pleiotrophic function, has been shown to provide an incremental clinical benefit in various patients, including very high-risk patients, such as those with acute coronary syndrome ${ }^{4,5)}$. A new technology utilizing proton nuclear magnetic resonance (NMR) has drawn more attention recently ${ }^{6}$. This new method uses signals emitted from methyl groups of lipids, yielding 16 lipoprotein subclass concentrations as well as particle numbers and sizes. Unfortunately, the effects of atorvastatin using this 
methodology in hypercholesterolemic subjects are largely unknown.

In the present study, we therefore attempted to investigate the effects of atorvastatin on lipoprotein subclass metabolism in hypercholesterolemic patients utilizing NMR methodology. We further assessed the potential effects of atorvastatin on inflammatory markers.

\section{Methods}

Twenty-six hypercholesterolemic patients were studied. Eligibility criteria included age between 40 to $75 \mathrm{yr}$, body mass index (BMI) less than $30 \mathrm{~kg} / \mathrm{m}^{2}$, and fasting LDL-C values $\geq 140 \mathrm{mg} / \mathrm{dL}$ both at screening and at the end of the run-in period. None of the study subjects had poorly controlled diabetes (defined as $\mathrm{HbA} 1 \mathrm{c} \geq 8 \%$ ) or evidence of thyroid, liver, or renal dysfunction and none were taking lipid-modifying medications. Four patients experienced effort angina. All subjects were instructed to maintain their diet, exercise, and alcohol intake throughout the study period. The study was approved by the Ethics Committee of Jikei University School of Medicine and study subjects gave written informed consent. After a 4-8 week run-in period, 26 patients received atorvastatin $10 \mathrm{mg}$ for 4 weeks. Fasting plasma was obtained prior to and at the end of the treatment, and then lipids, apolipoproteins, and other biochemical parameters, including remnant-like particles (RLP)-C, malondialdehyde-modified LDL (MDA-LDL), high sensitivity (hs) CRP, interleukin (IL)-6, and monocyte chemotactic protein (MCP) 1 were measured. Frozen plasma was shipped to LipoScience, Inc. (Raleiph, NC) for proton NMR analysis ${ }^{6}$, as we previously reported $^{7,8)}$. This new method is based on the signals emitted from methyl group protons of lipids, which were then calculated by the linear least-squares method to quantify lipoprotein subclass lipid concentrations (TG for VLDL, C for IDL, LDL, and HDL subclasses). Sixteen subclasses were simultaneously measured: six VLDL subclasses (VLDL6 $150 \pm 70 \mathrm{~nm}$, VLDL5 $70 \pm 10 \mathrm{~nm}$, VLDL4 $50 \pm 10 \mathrm{~nm}$, VLDL3 38 $\pm 3 \mathrm{~nm}$, VLDL2 $33 \pm 2 \mathrm{~nm}$, VLDL1 $29 \pm 2 \mathrm{~nm}), 4$ LDL subclasses (IDL $25 \pm 2 \mathrm{~nm}$, LDL3 $22 \pm 0.7 \mathrm{~nm}$, LDL2 $20.5 \pm 0.7 \mathrm{~nm}$, LDL1 $19 \pm 0.7 \mathrm{~nm}$ ), and $5 \mathrm{HDL}$ subclasses (HDL5 $11.5 \pm 1.5 \mathrm{~nm}$, HDL4 $9.4 \pm 0.6 \mathrm{~nm}$, HDL3 $8.5 \pm 0.3 \mathrm{~nm}$, HDL2 $8.0 \pm 0.2 \mathrm{~nm}$, HDL1 7.5 $\pm 0.2 \mathrm{~nm}$ ). For correlation analysis of NMR-defined lipoprotein subclasses with apolipoprotein, inflammation markers, large (VLDL5, 6), intermediate (VLDL3, 4 ), and small (VLDL1, 2) VLDL were employed. Likewise, large, intermediate, and small LDL and HDL represent LDL3 (HDL4, 5), LDL2 (HDL3), and
Table 1. Characteristics of study subjects and changes in lipids with atorvastatin

\begin{tabular}{|c|c|c|c|}
\hline & Baseline & Atorvastatin & Change (\%) \\
\hline $\mathrm{N}$ & 26 & & \\
\hline Age (yrs) & $54.7 \pm 12.5$ & & \\
\hline BMI $\left(\mathrm{kg} / \mathrm{m}^{2}\right)$ & $23.7 \pm 2.5$ & & \\
\hline Current smoker (\%) & $15 \%$ & & \\
\hline Hypertension (\%) & $35 \%$ & & \\
\hline Diabetes (\%) & $19 \%$ & & \\
\hline $\mathrm{TC}(\mathrm{mg} / \mathrm{dL})$ & $288 \pm 42$ & $198 \pm 31$ & $-31.5^{\ddagger}$ \\
\hline $\mathrm{TG}(\mathrm{mg} / \mathrm{dL})$ & $159 \pm 83$ & $115 \pm 59$ & $-27.4^{\dagger}$ \\
\hline HDL-C (mg/dL) & $61.8 \pm 25.1$ & $63.9 \pm 26.7$ & 3.4 \\
\hline LDL-C (mg/dL) & $195 \pm 41$ & $111 \pm 29$ & $-42.5^{+}$ \\
\hline RLP-C (mg/dL) & $14.3 \pm 9.5$ & $7.4 \pm 3.6$ & $-48.5^{\dagger}$ \\
\hline ApoA-I (mg/dL) & $157 \pm 43$ & $162 \pm 45$ & 2.8 \\
\hline ApoA-II (mg/dL) & $31.5 \pm 6.9$ & $31.5 \pm 7.8$ & 0.1 \\
\hline ApoB (mg/dL) & $137 \pm 38$ & $90 \pm 24$ & $-34.2^{\ddagger}$ \\
\hline ApoC-II (mg/dL) & $6.0 \pm 2.5$ & $4.5 \pm 1.9$ & $-25.0^{*}$ \\
\hline ApoC-III (mg/dL) & $16.3 \pm 6.9$ & $13.8 \pm 5.3$ & $-15.4^{\dagger}$ \\
\hline ApoE (mg/dL) & $5.4 \pm 1.0$ & $3.9 \pm 0.9$ & $-27.9^{\ddagger}$ \\
\hline
\end{tabular}

Values are the mean \pm SD, except percent values. ${ }^{\dagger} p<0.01{ }^{\ddagger} p<0.001$

LDL3 (HDL1, 2), respectively. LDL and HDL particle concentrations (nanomoles per liter) are calculated as the sum of LDL subclass concentrations, including IDL for the former and the sum of HDL subclasses for the latter. Average lipoprotein particle sizes were determined by weighting the mass percentage of each subclass by its diameter.

The apoE phenotype was determined by immunoblotting using a specific goat anti-apoE polyclonal antibody, as reported previously ${ }^{9}$.

Changes in biochemical parameters with atorvastatin treatment were analyzed by the paired t-test. Among lipid parameters, TG showed a skewed distribution, therefore; logarithmically transformed TG values were used for comparison. The distribution of LDL size (pattern A and B) was tested by the chisquare test. Pearson's correlation coefficients were calculated to examine the relationship between lipid parameters. A $p$ value $<0.05$ with a two-tailed test was considered significant. All statistical procedures were performed using SPSS software (version 9.1, SPSS Inc., Chicago, IL).

\section{Results}

Baseline characteristics of the study subjects, and lipid and lipoprotein concentrations at baseline and treatment are summarized in Table $\mathbf{1}$. The study subjects, consisting of 16 men and 8 women, were on 
Table 2. Effects of atorvastatin on lipoprotein subclasses parameters by NMR

\begin{tabular}{|c|c|c|c|}
\hline & Baseline & Atorvastatin & Change $(\%)$ \\
\hline \multicolumn{4}{|l|}{ VLDL (mg/dL triglyceride) } \\
\hline VLDL6 (large) & $4.9 \pm 9.6$ & $7.7 \pm 13.7$ & 57.7 \\
\hline VLDL5 & $30.7 \pm 44.2$ & $21.6 \pm 29.6$ & -29.5 \\
\hline VLDL4 & $50.8 \pm 34.4$ & $29.5 \pm 17.8$ & $-41.9^{\dagger}$ \\
\hline VLDL3 & $9.1 \pm 11.6$ & $5.8 \pm 7.6$ & -36.7 \\
\hline VLDL2 & $16.9 \pm 12.7$ & $3.7 \pm 3.6$ & $-78.3^{\text {米 }}$ \\
\hline VLDL1 (small) & $4.7 \pm 5.8$ & $6.2 \pm 4.2$ & 31.8 \\
\hline VLDL particle size (nm) & $44.3 \pm 7.7$ & $48.5 \pm 8.9$ & $9.7^{\dagger}$ \\
\hline \multicolumn{4}{|l|}{ LDL (mg/dL cholesterol) } \\
\hline IDL & $2.7 \pm 6.2$ & $1.9 \pm 3.0$ & -28.7 \\
\hline LDL3 (large) & $94.3 \pm 50.1$ & $67.0 \pm 33.0$ & $-28.9^{\text {क }}$ \\
\hline LDL2 & $26.9 \pm 24.6$ & $15.8 \pm 16.3$ & $-41.2^{\text {末 }}$ \\
\hline LDL1 (small) & $40.1 \pm 56.0$ & $18.9 \pm 32.2$ & $-53.6^{\text {क }}$ \\
\hline LDL particle size (nm) & $21.0 \pm 0.9$ & $21.2 \pm 0.8$ & $1.1^{\dagger}$ \\
\hline LDL particle number (nmol/L) & $1,802 \pm 496$ & $1,086 \pm 353$ & $-39.7^{\text {क }}$ \\
\hline \multicolumn{4}{|l|}{ LDL phenotype $^{\S}$} \\
\hline Pattern A (n) & 18 & 20 & \multirow{2}{*}{$p=0.53$} \\
\hline Pattern B (n) & 8 & 6 & \\
\hline \multicolumn{4}{|l|}{ HDL (mg/dL cholesterol) } \\
\hline HDL5 (large) & $14.6 \pm 19.8$ & $15.0 \pm 16.9$ & 2.8 \\
\hline HDL4 & $15.2 \pm 7.8$ & $19.9 \pm 7.4$ & $30.4^{*}$ \\
\hline HDL3 & $5.0 \pm 6.8$ & $6.0 \pm 6.0$ & 21.1 \\
\hline HDL2 & $21.3 \pm 8.0$ & $20.3 \pm 5.6$ & -4.6 \\
\hline HDL1 (small) & $1.9 \pm 3.5$ & $1.7 \pm 3.3$ & -12.0 \\
\hline HDL particle size $(\mathrm{nm})$ & $9.25 \pm 0.63$ & $9.34 \pm 0.55$ & $1.0^{*}$ \\
\hline HDL particle number $(\mu \mathrm{mol} / \mathrm{L})$ & $36.6 \pm 8.2$ & $37.8 \pm 8.0$ & 3.3 \\
\hline
\end{tabular}

average 55 years old and had an average BMI of 23.7 $\mathrm{kg} / \mathrm{m}^{2}$. The percentages of hypertension, diabetes, and current smokers were $35 \%, 19 \%$, and $15 \%$, respectively. Except for four patients, all were free from coronary artery disease evidenced by clinical symptoms, electrocardiogram, or laboratory data abnormalities. ApoE phenotypes were mostly E3/3, except for three subjects with E4/3 and one subject with E4/4. Plasma lipids and lipoproteins concentration at baseline and atorvastatin treatment are also listed in Table 1. The mean total cholesterol, TG, HDL-C, and LDL-C were $288,159,62$, and $195 \mathrm{mg} / \mathrm{dL}$, respectively, thus representing moderate to severe hypercholesterolemia. In addition to the marked reduction of TC $(-32 \%)$, LDL-C (-43\%), apoB ( $-34 \%)$, atorvastatin further decreased TG, RLP-C, apoC-II, apoC-III, and apoE by $27 \%, 49 \%, 25 \%, 15 \%$, and $28 \%$, respectively. In contrast, HDL-C, apoA-I, and apoA-II did not show significant changes by atorvastatin.
Effects on lipoprotein subclass parameters by NMR are summarized in Table $\mathbf{2}$. In general, atorvastatin reduced VLDL subclasses, in particular VLDL2 and VLDL4 concentrations, with a concomitant increase in VLDL1 and VLDL6. Overall, their alterations enlarged the average VLDL particle size. Although not significant, IDL decreased by $29 \%$, a finding consistent with the $49 \%$ reduction of RLP-C. Atorvastatin significantly reduced all LDL subclasses with greater \% reductions in smaller particles (LDL3 $-29 \%$, LDL2 - 41\%, LDL1 - 54\%), thus resulting in a significant reduction of LDL particle number $(1,802$ to $1,086 \mathrm{nmol} / \mathrm{L}, p<0.001)$ as well as a significant increase in the average LDL particle size (21.0 to $21.2 \mathrm{~nm}, p<0.05)$. The latter change, however, did not translate into a reduction of pattern $B$ relative to pattern A (31 to 23\%, $p=0.53$ ). Atorvastatin caused a modest effect on HDL subclass metabolism. A relatively large HDL, HDL4, significantly increased by 
Table 3. Correlation coefficient between $\%$ changes in LDL, HDL particle sizes, number and those in lipid parameters by atorvastatin

\begin{tabular}{lcccccc}
\hline & \multicolumn{3}{c}{ LDL } & & \multicolumn{2}{c}{ HDL } \\
\cline { 2 - 3 } \cline { 5 - 6 } & $\Delta$ LDL size & $\Delta$ LDL number & & $\Delta$ HDL size & $\Delta$ HDL number \\
\hline & & & & & & \\
$\Delta$ TC & -0.224 & $0.513^{\dagger}$ & & -0.172 & $0.513^{\dagger}$ \\
$\Delta$ TG (log) & -0.318 & 0.052 & & $-0.464^{*}$ & -0.288 \\
$\Delta$ LDL-C & -0.221 & $0.558^{\dagger}$ & & -0.010 & $0.525^{\dagger}$ \\
$\Delta$ HDL-C & 0.257 & -0.007 & & 0.386 & 0.361 \\
$\Delta$ RLP-C & $-0.639^{\dagger}$ & 0.418 & & $-0.585^{*}$ & 0.088 \\
$\Delta$ LDL size & - & $-0.684^{*}$ & & $0.672^{\ddagger}$ & -0.091 \\
$\Delta$ LDL number & - & - & & $-0.484^{*}$ & 0.231 \\
$\Delta$ HDL size & - & - & & - & -0.146 \\
\hline
\end{tabular}

$\Delta$, delta, ${ }^{*} p<0.05,{ }^{\dagger} p<0.01 \stackrel{\stackrel{\ddagger}{\ddagger}}{p}<0.001$

Table 4. Effects of atorvastatin on MDA-LDL concentration and inflammation markers

\begin{tabular}{lrcc}
\hline & Baseline & Atorvastatin & Change (\%) \\
\hline MDA-LDL (U/L) & $121.7 \pm 42.3$ & $79.5 \pm 31.6$ & $-34.7^{\text {* }}$ \\
MDA-LDL/apoB $\left(\mathrm{U} / \mathrm{mg} / 10^{-1}\right)$ & $1.05 \pm 0.86$ & $0.88 \pm 0.27$ & -16.2 \\
hsCRP $(\mathrm{mg} / \mathrm{L})$ & $0.66 \pm 0.42$ & $0.56 \pm 0.39$ & -15.5 \\
IL-6 (pg/mL) & $1.18 \pm 0.79$ & $0.87 \pm 0.72$ & $-26.5^{*}$ \\
MCP-1 $(\mathrm{pg} / \mathrm{mL})$ & $135.7 \pm 47.2$ & $144.3 \pm 50.3$ & 6.3 \\
\hline
\end{tabular}

Values are the mean $\pm \mathrm{SD}$.

${ }^{*} p=0.056,{ }^{*} p<0.01$

$30 \%$, thus resulting in a significant increase in the average HDL particle size ( 9.25 to $9.34 \mathrm{~nm}, p<0.05$ ).

Next, we correlated \% changes in LDL, HDL particle size and number with those in lipid parameters in order to gain insights into factors determining the sizes and numbers of these lipoproteins. As shown in Table 3, changes in LDL-C (TC) positively correlated with changes in the particle number of both LDL and HDL without any influence on particle size. Changes in remnants, represented by RLP-C, strongly and negatively correlated with particle size in both LDL and HDL. Changes in HDL-C seemed to positively correlate with changes in HDL size $(r=0.386$, $p=0.052)$ and number $(r=0.361, p=0.070)$. Changes in LDL size showed a strong and negative correlation with changes in LDL particle number, but a positive correlation with changes in HDL size. In contrast with LDL, there was no correlation between changes in size and number within HDL.

Effects of atorvastatin on inflammation markers are summarized in Table 4. MDA-LDL was significantly decreased by 35\%. Most inflammatory markers, except for MCP-1, showed modest to moderate non-significant reductions by atorvastatin. It is also worth mentioning that IL-6 showed a $27 \%$ reduction, which marginally failed to reach a statistically significant level.

Finally, we performed correlation analysis of \% changes in lipoprotein subclasses with those in apolipoproteins and inflammation markers. As summarized in Table 5, $\Delta$ apoA-I tended to correlate negatively with $\Delta$ small LDL $(p=0.07)$. $\Delta$ apoB was significantly and negatively correlated with $\Delta$ large and $\Delta$ intermediate VLDL, whereas positively with $\Delta$ small VLDL. $\Delta$ apoE showed an inverse correlation with $\Delta$ large HDL. Among inflammation markers, $\Delta$ hs-CRP was found to negatively correlate with $\Delta$ intermediate VLDL and $\Delta$ IL-6 positively with $\Delta$ small VLDL. Further, $\Delta$ MDA-LDL showed a strong, positive correlation with $\Delta$ small LDL.

\section{Discussion}

The present study is the first to investigate the effects of atorvastatin on lipoprotein subclass metabolism utilizing NMR methodology in hypercholesterolemic patients. Our NMR analysis demonstrated that atorvastatin markedly decreased all LDL subclasses, 
Table 5. Correlation coefficient between \% changes in NMR-defined lipoprotein subclass concentrations and those in apolipoproteins, inflammation parameters by atorvastatin

\begin{tabular}{|c|c|c|c|c|c|c|c|c|c|c|}
\hline & \multicolumn{6}{|c|}{ Apolipoproteins } & \multicolumn{4}{|c|}{ Inflammation markers } \\
\hline$\Delta$ large VLDL (25) & -0.105 & -0.114 & $-0.445^{*}$ & -0.083 & 0.025 & -0.069 & -0.216 & -0.133 & -0.043 & 0.280 \\
\hline$\Delta$ intermediate VLDL (26) & -0.327 & -0.167 & $-0.415^{*}$ & 0.148 & -0.228 & 0.148 & -0.382 & -0.293 & -0.099 & -0.162 \\
\hline$\Delta$ small VLDL (26) & -0.235 & -0.011 & $0.498^{*}$ & -0.031 & 0.001 & 0.202 & 0.014 & 0.246 & 0.423 & 0.081 \\
\hline$\Delta \mathrm{IDL}(10)$ & -0.415 & -0.200 & 0.279 & 0.039 & 0.062 & -0.254 & -0.504 & 0.087 & 0.146 & 0.537 \\
\hline$\Delta$ small LDL (17) & -0.485 & -0.157 & 0.213 & -0.322 & 0.084 & -0.217 & -0.200 & $0.760^{* *}$ & -0.324 & 0.034 \\
\hline$\Delta$ large HDL (26) & 0.183 & 0.011 & 0.019 & -0.176 & -0.173 & $-0.536^{*}$ & -0.042 & -0.036 & -0.032 & 0.136 \\
\hline$\Delta$ intermediate HDL (22) & 0.076 & 0.158 & 0.096 & 0.204 & 0.140 & 0.040 & 0.214 & -0.185 & -0.232 & 0.029 \\
\hline$\Delta$ small HDL (26) & 0.183 & 0.125 & -0.108 & 0.221 & -0.066 & -0.057 & -0.192 & -0.116 & 0.119 & -0.204 \\
\hline
\end{tabular}

Large, intermediate, and small VLDL comprise VLDL5 and 6, VLDL3 and 4, and VLDL1and 2. Likewise, large, intermediate, and small HDL comprise HDL 4 and 5, HDL3, and HDL1 and 2. Large, intermediate, and small LDL correspond to LDL3, LDL2, and LDL1.

Numbers in parentheses represents subject number after excluding subjects with a value of 0 either at baseline or with treatment.

$\Delta$, delta, ${ }^{*} p<0.05,{ }^{* *} p<0.05$

in particular, smaller LDL subclasses. This fraction, namely small dense LDL, is more atherogenic than other LDL subclasses due to its increased susceptibility to oxidation and glycation, resulting in lower binding capacity to the LDL receptor and retention in the extracellular matrix which is eventually taken up by macrophage via a scavenger receptor; therefore, it is conceivable that a qualitative change in favor of a shift to buoyant LDL, together with quantitative change (marked reduction in LDL particle number), by atorvastatin synergistically exerts a strong anti-atherogenic function. Furthermore, we found that some VLDL subclasses were decreased by atorvastatin treatment. This finding, together with the reductions of plasma TG, RLP-C, apoC-II, apoC-III, and apoE, can be interpreted as indicating that atorvastatin has a favorable effect on TG-rich lipoprotein metabolism. Although there are no previous studies to which our NMR data can be directly compared, the predominant reduction of dense LDL subclasses is supported by other NMR studies using patients with hypertriglyceridemia with ${ }^{10)}$ or without ${ }^{11)}$ hypercholesterolemia. Indeed, in vivo kinetic studies demonstrated the decreased production as well as increased clearance of VLDL, an observation consistent with the inhibition of VLDL secretion via the strong inhibition of HMG-Co reductase activity and increased direct uptake by an up-regulated LDL receptor ${ }^{12,13)}$.

Cardioprotective HDL is heterogeneous in size and apolipoprotein composition, which would, in turn, affect atherosclerosis differently; for example, large HDL, but not small HDL, is a predictor of cardiovascular risk ${ }^{14-16)}$. In this context, atorvastatin may exert a cardioprotective effect by increasing large HDL (HDL4).

Correlation analysis provides interesting insights into whether, if so how, changes in particle number and size in LDL and HDL associate with those in other lipid parameters. LDL and HDL size are negatively correlated with TG-rich lipoprotein metabolism, in particular RLP-C, demonstrating that improved TG-rich lipoprotein metabolism by atorvastatin results in the increased particle size of both LDL and HDL. Increased TG-rich lipoproteins cause increased CETPmediated lipid exchange with LDL which, in turn, becomes smaller with the hydrolysis of transferred TG by hepatic TG lipase. The parallel change between LDL and HDL size supports this mechanism since CETP-mediated lipid transfer of TG-rich lipoproteins should simultaneously occur with both LDL and HDL. Next, reduction of LDL-C was found to be significantly associated with the reduction of LDL and HDL particle numbers, but not size. Although the exact mechanisms for the reduction in HDL particle number along with LDL-C reduction are unclear, this may share a common mechanism by which a high dose of atorvastatin causes HDL reduction, thus deserving future study with a larger sample size. Among changes in NMR-defined lipoprotein subclasses, it is of note that $\Delta$ small LDL negatively associated with $\Delta$ apoA-I, again indicating metabolic linkage between LDL and HDL. $\Delta$ small LDL also positively correlated with $\triangle \mathrm{MDA}-\mathrm{LDL}$, thus supporting that MDA-LDL reasonably represents atherogenic small dense LDL metabolism.

Inflammatory markers, in particular IL-6, were 
significantly reduced by atorvastatin, demonstrating an anti-atherosclerotic effect additional to the potent lipid-lowering function.

There are some limitations to the present study. First, it lacks a placebo-control group to which the atorvastatin group can be directly compared. We therefore installed a run-in period to maintain the steady state throughout the study period. Nonetheless, a placebo-control study should be carried out to draw a solid conclusion in the future. Second, the treatment duration was relatively short (4 weeks). In this regard, our results are considered to be short-term effects which may be different from long-term effects. Third, our sample size may not be large enough to draw confident conclusions; thus, detailed analysis, including correlation analysis, should be interpreted with caution.

In summary, the results of the present study demonstrated that, in addition to its potent LDL-lowering effect, atorvastatin effectively improved atherogenic TG-rich lipoprotein profiles by reducing remnants, which, in turn, enlarged the LDL/HDL particle size. Further, some inflammation markers were inhibited by atorvatstatin treatment; therefore, atorvastatin may provide a benefit for hypercholesterolemic patients who are at increased risk for coronary heart disease.

\section{Acknowledgments}

This study was supported in part by Health and Labor Sciences Research Grants for Comprehensive Research on Aging and Health (H15-Chouju-012), Japan and a grant from Astellas Pharmaceutical Co., Ltd., Tokyo.

\section{References}

1) Expert panel on detectione, and treatment of high blood cholesterol in adults: Excutive summary of the the third report of the national cholesterol education program (NECP) expert panel on dectection, evaluation, and treatment of high bllod cholesterol in adults (adults teatment panel III). JAMA, 2001; 285:2486-2497

2) Pearson TA, Laurora I, Chu H, Kafonek S: The lipid treatment assessment project (L-TAP): a multicenter survey to evaluate the percentages of dyslipidemic patients receiving lipid-lowering therapy and achieving low-density lipoprotein cholesterol goals. Archives of Internal Medicine, 2000; 160:459-467

3) Vale MJ, Jelinek MV, Best JD, Health CsgCpoAC: How many patients with coronary heart disease are not achieving their risk-factor targets? Experience in Victoria 19961998 versus 1999-2000. Med J Aust, 2002; 176:211-215

4) Cannon CP, Braunwald E, McCabe CH, Rader DJ, Rouleau JL, Belder R, Joyal SV, Hill KA, Pfeffer MA, Skene
AM; Pravastatin or Atorvastatin Evaluation and Infection Therapy-Thrombolysis in Myocardial Infarction 22 Investigators: Intensive versus moderate lipid lowering with statins after acute coronary syndromes. N Engl J Med, 2004; 350:1495-1504

5) LaRosa JC, Grundy SM, Waters DD, Shear C, Barter P, Fruchart JC, Gotto AM, Greten H, Kastelein JJ, Shepherd J, Wenger NK, Treating to New Targets (TNT) Investigators: Intensive lipid lowering with atorvastatin in patients with stable coronary disease. $\mathrm{N}$ Engl J Med, 2005; 352:1425-1435

6) Otvos JD: Measurement of lipoprotein subclass profiles by nuclear magnetic resonance spectroscopy. Clinical Laboratory, 2002; 48:171-180

7) Ikewaki K, Tohyama J, Nakata Y, Wakikawa T, Kido T, Mochizuki S: Fenofibrate effectively reduces remnants, and small dense LDL, and increases HDL particle number in hypertriglyceridemic men - a nuclear magnetic resonance study. Journal of Atherosclerosis and Thrombosis, 2004; 11:278-285

8) Ikewaki K, Noma K, Tohyama J, Kido T, Mochizuki S: Effects of bezafibrate on lipoprotein subclasses and inflammatory markers in patients with hypertriglyceridemia-a nuclear magnetic resonance study. Int J Cardiol, 2005; 101:441-447

9) Kataoka S, Paidi M, Howard BV: Simplified isoelectric focusing/immunoblotting determination of apoprotein $\mathrm{E}$ phenotype. Clin Chem, 1994; 40:11-13

10) McKenney JM, McCormick LS, Schaefer EJ, Black DM, Watkins ML: Effect of niacin and atorvastatin on lipoprotein subclasses in patients with atherogenic dyslipidemia. Am J Cardiol, 2001; 88:270-274

11) Soedamah-Muthu SS, Colhoun HM, Thomason MJ, Betteridge DJ, Durrington PN, Hitman GA, Fuller JH, Julier K, Mackness MI, Neil HA, CARDS Investigators: The effect of atorvastatin on serum lipids, lipoproteins and NMR spectroscopy defined lipoprotein subclasses in type 2 diabetic patients with ischaemic heart disease. Atherosclerosis, 2003; 167:243-255

12) Mohammadi A, Macri J, Newton R, Romain T, Dulay D, Adeli K: Effects of atorvastatin on the intracellular stability and secretion of apolipoprotein B in HepG2 cells. Arterioscler Thromb Vasc Biol, 1998; 18:783-793

13) Parhofer KG, Barrett PH, Schwandt P: Atorvastatin improves postprandial lipoprotein metabolism in normolipidemlic subjects. J Clin Endocrinol Metab, 2000; 85:42244230

14) Asztalos BF, Roheim PS, Milani RL, Lefevre M, McNamara JR, Horvath KV, Schaefer EJ: Distribution of ApoA$\mathrm{I}$-containing HDL subpopulations in patients with coronary heart disease. Arterioscler Thromb Vasc Biol, 2000; 20:2670-2676

15) Gordon DJ, Rifkind BM: High-density lipoprotein--the clinical implications of recent studies. $N$ Engl J Med, 1989; 321:1311-1316

16) Stampfer MJ, Sacks FM, Salvini S, Willett WC, Hennekens $\mathrm{CH}$ : A prospective study of cholesterol, apolipoproteins, and the risk of myocardial infarction. $\mathrm{N}$ Engl J Med, 1991; 325:373-381 